\section{Thyroidectomy produces abnormal grooming behavior in cats*}

\author{
WALTER RANDALL and VIRGINIA PARSONS $\dagger$ \\ University of lowa, lowa City, lowa 52240
}

Cats with midbrain lesions exhibit an abnormal dissociation between appetitive and consummatory grooming behaviors and some signs of hypothyroidism. To determine if a hypothyroid state causes the abnormal dissociation, six cats were thyroidectomized and their behavior monitored. The thyroidectomized cats exhibited the same abnormal dissociation in grooming behavior as did the cats with midbrain lesions. However, certain considerations, including the latency and time course of the thyroidectomized-induced changes, suggest that the hypothyroid state is not directly involved, but rather brings about other endocrine and metabolic changes that are involved in the production of the behavioral abnormality.

During a study of abnormal grooming behaviors that were induced by lesions of the caudal midbrain region, histological signs of thyroid dysfunction were found (Randall, 1964). Subsequent investigations revealed that the grooming abnormality waxed and waned concomitantly with rhythmic fluctuations in thyroid activity (Randall \& Littschwager, 1967). The grooming abnormalities occur most frequently in the fall of the year, a time when the normal maximum in thyroid activity occurs. During the spring, a time of the normal troughs in thyroid activity, the cats with brain-stem lesions are normal, exhibiting none of the abnormal behavior that was present in the fall.

The abnormal grooming behavior, which appears after brain-stem lesions, consists of a dissociation between appetitive and consummatory grooming behaviors. The dissociation is detected by directing light tactile stimuli to the body surface of the cat. These light tactile stimuli, which have no effect on the normal cat, elicit three consummatory grooming behaviors. The particular consummatory behavior that is elicited depends on the part of the body surface that receives the tactile stimuli: Touch the head or neck or shoulder of the cat and a nonoriented nonfunctional scratching movement of the foot is elicited; stroke the lateral surface of the trunk and the tongue is protruded and a lick occurs; touch the dorsal surface of the body and a nondirected grooming bite occurs in midair. These three consummatory behaviors occur in normal cats at the end of orienting behaviors but are elicited in lesioned cats by tactile stimuli without the normally preceding appetitive components.

*This research was supported by Grant MH 15402-03. from the National Institute of Mental Health, United States Public Health Service.

†Now at the University of Wisconsin-Parkside 53140 .
The measure of thyroid activity which varied concomitantly with the abnormal grooming behavior was the quantity of a tracer dose of 131I excreted in the urine. This measure was used because of the ease of obtaining repeated measures throughout the year with only minor disturbances to the cats. But the urinary excretion of an injected dose of $131 \mathrm{I}$ is not necessarily a very specific measure of thyroid activity because the measure may readily be influenced by the amount of dietary iodide and by kidney function. Thus, a more specific measure of thyroid activity, the rate of release of thyroid hormone from the thyroid gland, was used to validate the $131 \mathrm{I}$ excretion measures. The rate of release of hormone from the gland was estimated by counting the gamma irradiation from the 131 l-labeled thyroid gland on successive days. The rate-of-release measure supported a thyroid-activity interpretation of the excretion scores, and, in addition, a difference in thyroid activity between normal and lesioned cats was detected, a difference not present in the $131 \mathrm{I}$ excretion measures. The lesioned cats were hypothyroid. This group difference in thyroid activity was supported by other measures; measurements made of the histological features of the thyroid glands indicated that the lesioned group possessed larger follicles and flatter epithelia cells than the normal cats; also, the fecal excretion of $131 \mathrm{I}$ was less in the lesioned group. Thus, several different measures of thyroid activity indicated that the lesioned group was hypothyroid and suggested that hypothyroidism might be involved in the genesis of the abnormal grooming behavior.

In the present study several cats were thyroidectomized and their behavior monitored to see if they would exhibit the same abnormalities that are found in cats with brain-stem lesions. A further step in assigning behavioral changes in the thyroidectomized cats to the absence of thyroid hormones involved attempts to reverse any changes by administering thyroid hormones.

\section{METHOD}

Six adult male cats were thyroidectomized. The thyroidectomy was accomplished with ablation dosages of 1311, the best method because thyroid tissue is destroyed regardless of its locus, and because the parathyroid tissue is spared. The method and the dosages were obtained from Crawford (1961), who used cats and included histological verifications of the thyroid ablations at the termination of his study. Four millicuries of 1311 were injected intramusculary into each cat; a similar injection was given a month later.

Fourteen cats received stereotaxic lesions of the caudal midbrain region. Details of the method and anatomical reconstruction of similar lesion groups have been presented (Randall et al, 1967). Ten normal cats were used as controls.

Both before and after thyroidectomy and before and after midbrain lesions, the cats' body surfaces were monitored for elicitable grooming fragments. Light tactile stimuli were directed to the head and neck and to the dorsal and lateral surfaces of the trunk, and the occurrence of nonfunctional and nonoriented consummatory grooming behaviors was recorded. The body surface of the control group was monitored also.

When abnormal grooming behaviors appeared in the thyroidectomized cats, thyroid hormones were administered in an effort to restore the cats to a normal state. The cats received intramuscular injections of a mixture of T3 (Liothyronine sodium, L-form, "Cytomel" from Smith, Kline, and French Laboratories) and T4 (tetraiodothyronine, L-form, from Sigma Chemical Company). The thyroid hormone mixture was dissolved in .01N $\mathrm{NaOH}$ and diluted with physiological saline to a final concentration of $.04 \mathrm{mg} / \mathrm{ml}$ T3 and $.06 \mathrm{mg} / \mathrm{ml} \mathrm{T} 4$. Thus, each milliliter of the mixture contained $.1 \mathrm{mg}$ of thyroid hormones, with the $\mathrm{T} 4$ to $\mathrm{T} 3$ ratio $3: 2$. The mixture was then sterilized in an autoclave for $30 \mathrm{~min}$. A new mixture of the same concentration was made and autoclaved each week. Autoclaving destroys some fraction of the thyroid hormones; so, in order to determine the proper dosages for maintaining a euthyroid state, a group of normal cats was injected with the thyroid hormone mixture and their body weight was monitored. After 2 weeks of daily injections of $1 / 2 \mathrm{ml}$ of the mixture, the normal cats began losing weight, and the dosage was cut back to an average daily injection of $4 \mathrm{ml}$. At this dosage level, the normal cats maintained their body weight, so the thyroidectomized 
cats were injected intramuscularly at this dosage level. T3-T4 replacement therapy was continued for 2 months. Both T3 and T4 were used because of the lack of information on thyroid hormones in the cat.

\section{RESULTS}

Consummatory grooming fragments became elicitable in the thyroidectomized cats and in the cats with lesions of the caudal midbrain region. The abnormality in grooming behavior in the thyroidectomized cats was the same abnormality as that in the cats with lesions of the caudal midbrain region. When the thyroidectomized cats were administered thyroid hormones, they became normal.

After thyroidectomy, several months elapsed before any grooming fragments were detectable. The first ablation dose of $131 \mathrm{I}$ was administered in March, and the first appearance of a consummatory fragment occurred in the last week of May. By July, five of the six thyroidectomized cats were exhibiting grooming fragments, and the sixth cat never consistently exhibited grooming fragments until March of the following year. This time course for the initial appearance of the grooming fragments was similar to the time course in brain-stem-lesioned cats. Another similarity of the abnormal grooming behavior in the thyroidectomized cats to the brain-stem-lesioned cats was the time course of the spontaneous and repeated reversions between the normal grooming state and the state where consummatory fragments may be elicited, the two groups exhibiting onsets and offsets of the abnormal grooming state synchronously. For both groups the highest frequency of elicitable grooming fragments occurred in the fall of the year, with both groups approaching a normal condition in the spring. Other minor fluctuations occurred, so that a rhythm of 4 months" duration existed (see, e.g., Randall \& Parsons, 1969). The abnormal grooming state and other signs of thyroidectomy (skin lesions, abnormally scaly and oily skin, loss of pelage, swollen abdomen, hoarseness of voice, and a black pigmentation of the dorsal surface of the nose) remitted gradually with the daily injections of thyroid hormones, requiring 2 months of daily injections before the normal state was obtained.

No signs of thyroidectomy were evident in the midbrain-lesioned group, except the abnormal grooming behavior.

\section{DISCUSSION}

The simplest way to account for the same abnormal behavior from both midbrain lesions and thyroidectomy is by assuming that the midbrain lesion brings about a hypothyroid state. But four considerations made this simplest possibility unlikely. First, cats with midbrain lesions do not exhibit clear and univocal signs of hypothyroidism. None of the obvious signs of a hypothyroid state, like voice or pelage changes, occurs in midbrain-lesioned cats, and even though some measures of thyroid activity obtained from the lesioned cats are indicative of a depressed level of thyroid activity, other measures of thyroid activity fall within the normal range. Second, the reversions of the behavioral abnormality occur in the thyroidectomized cats with a time course in phase with the same abnormality in the lesioned cats; thus, the thyroid gland itself is eliminated from consideration as the source of the spontaneous reversions. Third, because of the long latency of the behavioral changes following thyroidectomy and thyroid hormone replacement, it is difficult to consider thyroid hormones alone as important determinants of the abnormal grooming state. Rather, a latency of many weeks suggests that both midbrain lesions and thyroidectomy bring about profound metabolic changes, and these complex changes in the milieu, or some aspect of them, may be the bases of the grooming abnormalities. Fourth, the midbrain is implicated as part of the control circuitry for the regulation of systemic levels of adrenocortical hormones, whereas evidence for a thyroid function of the midbrain is sparse. Anderson et al (1957) reported a change in thyroid function following complete transection of the brain stem at an intercollicular level, but the study is difficult to evaluate; most of the animals lived only a few days after the surgery, and there were major changes in adrenocortical function. Thus, the change in thyroid state could have been a morbid condition or a state compensatory to a lesion-induced primary change in the adrenocorticoids. A study with rats by Beattie \& Chambers (1953) reported a lowered oxygen consumption following lesion of the brain stem, but the lesions were in and around the habenular nuclei, apparently confined to the pretectal area, and not encroaching on the ventral-lateral parts of the midbrain tegmentum. The evidence for midbrain control of systemic levels of adrenocorticoids is direct; cortisone implanted into the midbrain depresses systemic levels of adrenocortical hormones (Endroczi et al, 1961), a finding that has been replicated in other laboratories (e.g., Corbin et al, 1965). Some conceptual difficulty exists because the existence of negative-feedback sites in the hypothalamus or pituitary is well established: e.g., in the recent hypothalamic island studies (Halász, 1969), the changes in systemic levels of adrenocorticoids appropriate to an intact negative-feedback system are obtained with the hypothalamus and pituitary together isolated from the remainder of the central nervous system. However, the level of adrenocorticoids is elevated merely as a result of the separation of the hypothalamus and pituitary from the rest of the brain, and this elevated level could exist because of the absence of additional negative-feedback functions of the midbrain.

If negative-feedback sites for adrenocortical hormones are destroyed by the midbrain lesion, a malfunctioning control system could generate abnormal adrenocorticoid rhythms that, under certain milieu conditions present at certain times of the year, may contribute essentially to the physiological bases of the abnormal grooming behavior. Interrelationships of thyroid and adrenocortical hormones are well known [e.g., in thyroidectomized rats degeneration of the adrenal cortex occurs (Money, 1955)], so that the absence of a normal thyroid rhythm in thyroidectomized animals may result in abnormal rhythms in adrenocortical hormones.

\section{REFERENCES}

ANDERSON, E., BATES, R., HARTHORNE, E., HAYMAKER, W., KNOWLTON, K., RIOCH, D., SPENCE, W., \& WILSON, H. The effects of midbrain and spinal cord transection on endocrine and metabolic functions with postulation of a midbrain hypothalamico-pituitary activating system. In G. Pincus (Ed.), Recent progress in hormone research. Vol. XIII, New York: Academic Press, Inc., 1957. Pp. 21-57.

BEATTIE, J., \& CHAMBERS, R. Changes in oxygen consumption and radioiodine $\left({ }^{131} 1_{1}\right)$ uptake following hypothalamic lesions in the albino rat. Quarterly Journal of Experimental Physiology, 1953, 38, 75-84.

CORBIN, A., MANGILI, G., MOTTA, M., \& MARTINI, L. Effect of hypothalamic and mesencephalic steriod implantation on ACTH feedback mechanisms. Endocrinology, 1965, $76,811-818$.

CRAWFORD, J. W. Visual flicker fusion frequency and thyroid function in the cat. Unpublished $\mathrm{PhD}$ dissertation, University of Chicago, 1961.

ENDROCZI, E., LISSAK, K., \& TEKERES, M. Hormonal "feed-back" regulation of pituitary-adrenocortical activity. Acta Physiology (Hungary), 1961, 18, 291-299.

HALÁSZ, BELA. The endocrine effects of isolation of the hypothalamus from the rest of the brain. In W. F. Ganong and L. Martini (Eds.), Frontiers in neuroendocrinology. New York: Oxford University Press, 1969. Pp. 307-342.

MONEY, W. The interrelation of the thyroid and the adrenals. In The thyroid. Upton, New York: U. S. Brookhaven National Laboratory, 1955. Pp. 137-168.

RANDALL, W. The behavior of cats (Felis catus $L$.) with lesions in the caudal midbrain region. Behaviour, 1964, 23, 107-139. 
RANDALL, W.. LAKSO. V.. \& LIITTSCHWAGER. J. Lesion-induced dissociations between appetitive and consummatory grooming behaviors and their rilationship to body weight and food intake rlsythms. Journal of Comparative and Physiological Psychology. 1969. 68, 476-483.

RANDALI. W.. \& LIITTSCHWAGER. J. The relationship between cycic changes in thyroid tunction and behavior of cats with brain stem lesions. Journal of Psychiatric Research. 1967. 5. 39-58.

RANDALI. W.. \& PARSONS, $V$. The concomitancy in the rhythms of caloric intake and behavior in cats: A replication. Psychonomic Science, 1969. 15. 35-36.

\title{
The behavior of clouds correlates with thyroid activity in cats
}

\author{
WAL TER RANDALL \\ University of Iowa. Iowa City. lowa 52240
}

Three- to 5-month rhythms in a lesion-induced abnormal grooming behavior and thyroid activity of cats have been found. An analysis of the climatological data for the same period revealed similar rhythms in sunshine. The sunshine peaks preceded the thyroid activity peaks, and a cross correlation was significant with a temporal lag of 1 month.

Cats in the laboratory with year-round air conditioning, with the temperature and humidity controlled so that no seasonal changes may occur, display 3- to 5-month rhythms in thyroid activity (Randall \& Liittschwager, 1967). Caloric intake also exhibits similar long-term rhythms of 3 to 5 months duration, and in cats with brain-stem lesions. an abnormal grooming behavior waxes and wanes concomitantly with these rhythms (Randall. Lakso, \& Liittschwager, 1969). And both normal and lesioned cats exhibit a prominent annual rhythm in adrenalin and noradrenalin excretion (Parsons, 1970).

An interesting aspect of these rhy thms is the synchrony among the groups from different years, as well as the synchrony among individuals within a group. Many routine things are occurring in the laboratory that could provide an external synchronizer for the hormone rhythms. For example, the lights in the colony are never on except during daylight so that the normal patterns of environmental lighting are obtained through the windows. Thus, the annual rhythms may be synchronized by lights or by our light-controlled activity. However, a possible external synchronizer for the approximately 4-month rhythms is not readily evident from a consideration of seasonal events. A search of the current review literature on biological rhythms and their synchronizers (e.g., Sollberger, 1965) failed to reveal any environmental, climatological, or astronomical event that varies with a 3- to 5-month period. However, other 3- to 5- month rhythms were found: activity cycles in rats, and the occurrences of epileptic fits, peptic ulcers, and periodic catatonia in humans. King (1940) found four peaks during a year in

the iodine content of human thyroid glands.

Finally our attention was directed to climatological data because of the "sunshine curves" of Wade H. Brown, which he published in his Harvey Lecture (1928). Brown did an exhaustive series of experiments on organ weights, blood calcium, and blood phosphorus on over 600 rabbits during a $3 \frac{1}{2}$-year period in the 1920s. His persistent and insightful concern with this unwieldy mass of data led to the discovery that organ weights and calcium and phosphorus concentrations varied systematically with time. In an effort to account for the prominent annual rhythms in his smoothed averages, Brown smoothed 2 years of data on percentage of possible sunshine. The sunshine curves, which were considered "distinctly abnormal" by Brown, show 3-month rhythms, with the four peaks and troughs occurring during the same months for each year. In the present study, sunshine data obtained from the weather stations around the site of a study of behavior and thyroid activity in cats were analyzed with a periodic regression technique. The multiple peaks and troughs during the year which Brown considered atypical were found and were correlated with thyroid activity.

\section{METHOD}

Monthly values of percentage of possible sunshine, the percentage of time between sunrise and sunset when no clouds are between the sun and a recording device, were obtained from the U.S. Weather Bureau and analyzed with a periodic regression technique. The periodic regression analysis was developed by Bliss (1958) and consists of curve fitting with the trigonometric fitting functions of the Fourier series, followed by an analysis of variance to evaluate the fitted curves. The mathematical model, then. is

$$
y_{t}=a_{o}+\sum_{i=1}^{j}\left(a_{i} \cos \frac{2 \pi i t}{k}+b_{i} \sin \frac{2 \pi i t}{k}\right),
$$

where $y_{t}$ is the derived value for time $t, a_{0}$ is the overall mean. $k$ is the number of repeated measures $(k=12$ in this study), $j$ is the index of the last harmonic of the series $(j=k / 2)$, and $a_{i}$ and $b_{i}$ are the coefficients that are derived with a least-squares criterion. Complete details and illustrative examples of these procedures are presented by Bliss (1958). Briefly, the Fourier coefficients, $a_{i}$ and $b_{i}$, are computed with a least-squares criterion, and different Fourier curves, one for each addition of a harmonic to the series, are plotted. Then the analysis of variance is used to determine if one of the family of Fourier curves is an acceptable representation of the data.

The Fourier series was fitted to a year of percentage-possible-sunshine data from 12 weather stations, one measure per month from each station. The 12 weather stations were within a 240-mile radius of Durham, North Carolina, the site where thyroid activity and behavioral data were obtained on cats (Randall et al, 1967). The 240-mile radius was selected merely to include a suitable number of stations for the statistical analysis. Six of the stations were in North Carolina: Raleigh, Greensboro, Charlotte, Wilmington. Cape Hatteras, and Asheville; three of the stations were in Virginia: Lynchburg, Richmond, and Norfolk; and three of the stations were in South Carolina: Columbia, Greenville, and Charleston.

A Pearson product-moment correlation coefficient was obtained for the sunshine and thyroid data, with a temporal lag of 1 month in the sunshine data.

\section{RESULTS}

Figure 1 shows the least-squares fitted curves for percentage possible hours of sunshine and for thyroid activity in 10 normal cats. A 4-month rhythm exists for both variables. The sunshine peaks precede the thyroid activity peaks, and with a temporal lag of 1 month in the sunshine data, the Pearson product-moment correlation coefficient is $0.80(p<0.01)$. The correlation coefficient was obtained on the monthly means from the 12 weather stations and the monthly means of the thyroid activity of the 10 normal cats. Table 1 is a summary of the analysis of variance of the sunshine data, indicating that a significant mean square was assigned to the first three fitting functions (Rows 2 , 